LA GRANJA:

REVISTA DE

CIENCIAS DE LA VIDA

pISSN:1390-3799; eISSN:1390-8596

http:/ / doi.org/10.17163/lgr.n29.2019.07
Artículo científico / Scientific paper

MANEJO PECUARIO

\title{
EDAD AL PRIMER PARTO E INDICADORES DE EFICIENCIA EN VACAS LECHERAS CON DIFERENTE POTENCIALIDAD PRODUCTIVIDAD EN SISTEMAS A PASTOREO
}

\author{
AGE AT THE FIRST CALVING AND EFFICIENCY INDICATORS IN DAIRY COWS \\ WITH DIFFERENT PRODUCTIVE POTENTIAL IN GRAZING SYSTEMS
}

\author{
Pablo Roberto Marini*1,2,3 ${ }^{\text {[D }}$ y Ricardo José Di Masso ${ }^{2,3}$
}

${ }^{1}$ Cátedra de Producción de Bovinos de Leche. Facultad de Ciencias Veterinarias. Universidad Nacional de Rosario. Argentina

${ }^{2}$ Centro Latinoamericano de Estudios de Problemáticas Lecheras (CLEPL). Facultad de Ciencias Veterinarias, Universidad Nacional de Rosario, Argentina

${ }^{3}$ Carrera del Investigador Científico CIC-UNR, Universidad Nacional de Rosario, Argentina

*Autor para correspondencia: pmarini@unr.edu.ar

Manuscrito recibido el 15 de agosto de 2018. Aceptado, tras revisión, el 11 de febrero de 2019. Publicado el 1 de marzo de 2019.

\section{Resumen}

El objetivo del presente trabajo fue analizar la relación entre la edad al primer parto con la producción láctea e indicadores reproductivos en vacas lecheras, con diferente potencial productivo. Se utilizaron datos retrospectivos de 260 vacas de raza Holstein biotipo Americano-Canadiense con información desde su nacimiento hasta finalizar la lactancia, recolectados entre los años 1992-2012, en el tambo cabaña perteneciente a la Escuela Agrotécnica Gral. San Martín dependiente de la Universidad Nacional de Rosario, ubicado en la localidad de Casilda, Departamento Caseros, provincia de Santa Fe, Argentina. Los animales se dividieron en dos categorías: vacas puras (n=103) y vacas con registro de cría $(\mathrm{n}=157)$. Cada una de las vacas pertenecientes a cada uno de los grupos mencionados se caracterizó en función de los valores de dos indicadores reproductivos: edad al primer parto en días e intervalo, parto concepción en días y dos indicadores productivos: producción de leche ajustada a 305 días de lactancia en litros y número de partos. No hubo diferencias significativas entre grupos en su precocidad. Las vacas puras presentaron mayor producción $(t=4,493 ; P<0,0001)$ mientras que las vacas con registro de cría fueron más fértiles $(t=2,039 ; P=0,043)$. Los grupos no difirieron en el número de partos. Las diferencias en el comportamiento de las vacas puras y las vacas con registro de cría con diferente potencialidad productivo ponen en discusión el objetivo de ejercer presión para lograr un primer parto a los 24 meses como propuesta generalizada en los sistemas a pastoreo.

Palabras clave: Producción de leche, reproducción, longevidad, eficiencia. 
The objective of this study was to analyze the relationship between age at first calving with dairy production and reproductive indicators in dairy cows with different productive potential. Retrospective data of 260 Holstein AmericanCanadian biotype cows with information from birth to the end of lactation, collected from 1992 to 2012 in the dairy farm belonging to the agro-technical school Gral. San Martín, National University of Rosario, were used. The farm is located in the city of Casilda, province of Santa Fe, Argentina. Cows were divided into two categories: pure cows (n $=103$ ) and cows with breeding records $(n=157)$. Each cow belonging to each of the aforementioned groups was characterized according to the values of two reproductive indicators: age at first calving in days and calving-conception interval in days and two productive indicators: milk production adjusted to 305 days of lactation in liters, and number of calving. There were no significant differences between groups in age at first calving. The pure cows presented higher production $(t=4,493, P<0,0001)$ while the cows with breeding record were more fertile $(t=2,039, P=0,043)$. Groups did not differ in the number of calving. Differences in the behavior of pure cows and cows with breeding records with different productive potential puts into discussion the objective of exerting pressure to achieve a first delivery at 24 months as a generalized proposal in the grazing systems.

Keywords: Milk production, reproduction, longevity, efficiency.

Forma sugerida de citar: Pablo Roberto Marini y Ricardo José Di Masso (2019). Edad al primer parto e indicadores de eficiencia en vacas lecheras con diferente potencialidad productividad en sistemas a pastoreo. La Granja: Revista de Ciencias de la Vida. Vol. 29(1):84-96. http://doi.org/10.17163/lgr.n29.2019.07.

IDs Orcid:

Pablo Roberto Marini: http:/ / orcid.org/0000-0003-0826-0387

Ricardo José Di Masso: https:/ /orcid.org/0000-0003-4873-5156 


\section{Introducción}

La edad al primer parto es un indicador del tiempo que tarda un animal en alcanzar su madurez sexual y reproducirse por primera vez (Hare, Norman y Wright, 2006); la misma puede verse influenciada por el tamaño corporal y el inicio de la actividad hormonal del sistema reproductivo (Moore y col., 1991). Aunque se considera que la edad a la pubertad no está determinada por un peso corporal en particular, sí lo está por un orden indeterminado de condiciones fisiológicas que resultan de un peso dado (Grajales, Hernández y Prieto, 2006). En los sistemas productivos en pastoreo, la relación peso corporal-inicio de la actividad reproductiva se hace más extrema (Marini, Charmandarian y Di Masso, 2007). Debido a la corta vida productiva de las vacas lecheras (Novaira y col., 2018; Seegers y col., 1998), se requiere de una eficiente crianza de las vaquillonas destinadas a reemplazar a las vacas descartadas. Las etapas de cría y recría de esas vaquillonas son períodos improductivos, en donde el ingreso por venta de las vacas descartadas sólo aporta al mantenimiento de la alimentación de la vaca que ingresa a producción, por lo que los gastos de las etapas previas no se recuperan. La crianza de las hembras de reposición dura al menos dos años (Hare, Norman y Wright, 2006) lapso que, en la actualidad, equivale a casi la mitad de la vida útil de las vacas lecheras. En este contexto, aumentar la longevidad de las vacas permitiría diluir el impacto de la etapa no productiva (cría y recría) con una mayor productividad (Grandl y col., 2016).

A este contexto se suma que la recría de vaquillonas es una inversión que no genera retorno hasta después de un parto o una venta y es por esto que se le presta atención en los primeros meses de vida de la ternera (Pieroni, 2014). Es importante evaluar las implicaciones económicas que tiene esta situación sobre la empresa lechera, donde una categoría totalmente improductiva se mantiene en el sistema por mayor tiempo. La vaquillona de reposición representa aproximadamente entre el 15 y $20 \%$ de los costos operativos del tambo y se considera como el tercer costo después de la alimentación y la mano de obra en nuestros establecimientos lecheros (Berra, 1998). Marini, Charmandarian y Oyarzabal, (2001) en base a registros de 1282 vacas de primera lactancia provenientes de cinco establecimientos lecheros en sistemas a pastoreo con diferentes niveles de suplementación observaron que la edad al pri- mer parto no afectó en forma significativa los valores de dos indicadores productivos (producción de leche y duración de la lactancia) y cuatro indicadores reproductivos (intervalo parto primer celo, intervalo parto primer servicio, intervalo parto concepción y número de servicios por preñez). De hecho, la tasa de reemplazo anual es alta en la mayoría de los países y el número promedio de lactaciones en el momento del refugo es generalmente bajo, alrededor de tres en Francia y de similares valores en Perú (Seegers y col., 1998; Orrego, Delgado y Echevarría, 2003). Hadley, Wolf y Harsh, (2006) observaron una tasa de sacrificio promedio que varía entre $30 \%$ y $40 \%$ en hatos lecheros de los Estados Unidos, cuando la tasa óptima de sacrificio a nivel de rebaño debe variar de $19 \%$ a $29 \%$. El objetivo del presente trabajo fue analizar la relación entre la edad al primer parto con la producción láctea e indicadores reproductivos en vacas lecheras de primera lactancia, con diferente potencial productivo y mantenidas en sistemas a pastoreo.

\section{Materiales y métodos}

Se utilizaron datos retrospectivos correspondientes a las lactancias de 260 vacas primíparas de raza Holstein biotipo Americano-Canadiense con información desde su nacimiento hasta finalizar la lactancia, recolectados entre los años 1992-2012 en el tambo cabaña perteneciente a la Escuela Agrotécnica Gral. San Martín dependiente de la Universidad Nacional de Rosario. El mismo se encuentra ubicado en la localidad de Casilda, Departamento Caseros, provincia de Santa Fe, Argentina $\left(33^{\circ} 2^{\prime} 39^{\prime \prime}\right.$ de latitud sur, $61^{\circ} 10^{\prime} 5^{\prime \prime}$ de longitud oeste), área que cuenta con control lechero de la Sociedad Rural de Totoras, Entidad Oficial $N^{\circ} 13$ y presenta las siguientes características: (1) utiliza exclusivamente vacas de la raza Holstein biotipo AmericanoCanadiense, (2) la alimentación es básicamente a pastoreo (praderas de alfalfa) con suplementación (grano de maíz, silo de maíz y rollos) suministrada en diferentes proporciones de acuerdo a la disponibilidad estacional de las praderas de alfalfa, (3) se cumple con un control ginecológico periódico, (4) se realiza control lechero oficial, (5) se encuentra libre de brucelosis, tuberculosis, campilobacteriosis y tricomoniasis; con control de leptospirosis, rinotraqueitis infecciosa bovina y diarrea viral bovina, (7) se insemina artificialmente con semen de origen 
americano y canadiense. En el lapso relevado, todas las vacas se manejaron en las mismas instalaciones de ordeño. Los animales se dividieron en dos categorías: vacas puras (VP) $(\mathrm{n}=103)$ y vacas con registro de cría (VRC) $(n=157)$. La diferencia entre ambas se basa en que las primeras se inseminan siempre con semen de toros probados, mientras que dicha práctica no se mantiene de manera constante en el caso de las segundas. Para alcanzar la condición de vaca pura se requieren siete generaciones con padres probados, lo que implica que las integrantes del grupo de vacas con registro de cría están en distintos momentos de ese camino para lograrlo.

Durante el período objeto de esta evaluación las vacas consumieron forrajes bajo pastoreo directo (pasturas polifíticas y verdeos anuales de invierno y de verano) o conservados (silo de planta entera de maíz y de sorgo, henos de praderas) y concentrados (granos de maíz y de sorgo). El ambiente climático durante el mismo fue muy variable, tanto en precipitaciones como en la combinación de temperatura y humedad relativa del ambiente. Cada una de las vacas pertenecientes a cada uno de los dos grupos mencionados se caracterizó en función de los valores de dos indicadores reproductivos: edad al primer parto en días (EPP) y el intervalo parto- concepción en días (IPC), y dos indicadores productivos: producción de leche ajustada a 305 días de lactancia en litros (PL) y longevidad (número de partos, NP). Las vacas de cada grupo se dividieron en cuatro subgrupos por su edad al primer parto, tomando como criterio los valores del cuartil de primer orden, la mediana y el cuartil de tercer orden. El efecto del grupo de pertenencia sobre cada una de las variables se evaluó con un análisis de la varianza a un criterio de clasificación, seguido de la prueba $t$ de Student a excepción de la supervivencia (número de partos) para la cual se utilizó el análisis de la varianza por rangos de Kruskal-Wallis y la prueba de comparaciones múltiples de Dunn.

Para el análisis entre cuartiles se utilizó un análisis de la varianza a un criterio de clasificación, seguido de la prueba de comparaciones múltiples de Tukey, a excepción de la supervivencia (número de partos) para la cual se utilizó el análisis de la varianza por rangos de Kruskal-Wallis y la prueba de comparaciones múltiples de Dunn. Las asociaciones entre variables se cuantificaron a partir del cálculo del coeficiente de correlación lineal producto-momento de Pearson o del coeficiente de correlación no paramétrico de Spearman según correspondiera.

Tabla 1. Indicadores productivo-reproductivos en vacas lecheras con diferente potencial de producción en sistemas a pastoreo.

\begin{tabular}{|c|c|c|}
\hline & Vacas puras & Vacas con registro de cría \\
\hline${ }^{1}$ Precocidad & $964,9 \pm 15,54$ & $980,4 \pm 15,47$ \\
\hline${ }^{1}$ Producción & $5835 \pm 138,3$ & $5134 \pm 87,9$ \\
\hline${ }^{1}$ Fertilidad & $196,3 \pm 9,08$ & $172,9 \pm 7,14$ \\
\hline${ }^{2}$ Supervivencia & $3(1-8)$ & $3(1-10)$ \\
\hline \multicolumn{3}{|c|}{$\begin{array}{l}1 \text { Valores: media aritmética } \pm \text { error estándar. Prueba } t \text { de Student para } \\
\text { datos independientes. Precocidad: Edad al primer parto en días, Pro- } \\
\text { ducción: litros totales al finalizar la primera lactancia ajustada a } 305 \\
\text { días. Fertilidad: intervalo parto-concepción. } \\
2 \text { Valores: mediana (rango). Prueba } U \text { de Mann-Whitney. Supervivencia: } \\
\text { número de partos en su vida productiva. }\end{array}$} \\
\hline
\end{tabular}

\section{Resultados}

La Tabla 1, muestra ausencia de diferencias estadísticamente significativas entre grupos en su precocidad $(t=0,675 ; P=0,500)$. Las vacas puras presentaron mayor producción de leche $(t=4,493 ; P<$ $0,0001)$ mientras que las vacas con registro de cría fueron más fértiles $(t=2,039 ; P=0,043)$. Los grupos no difirieron en su supervivencia $(U=7264 ; P=$
$0,159)$, aunque se observaron vacas con mayor número total de partos en comparación con aquellas con registro de cría. La Tabla 2 muestra las diferencias generadas al categorizar a las vacas de ambos grupos por cuartiles de acuerdo a su edad al primer parto. Las vacas ubicadas en el primer cuartil con una edad cercana a los 25 meses presentaron valores óptimos de precocidad. Aquellas ubicadas en los 
Tabla 2. Edad al primer parto (días) de vacas lecheras con diferente potencial de producción, discriminadas en cuartiles.

\begin{tabular}{cccc}
\hline \multicolumn{4}{c}{ Vacas puras } \\
\hline Cuartil 1 & Cuartil 2 & Cuartil 3 & Cuartil 4 \\
\hline $771,8 \pm 17,27^{a}$ & $920,0 \pm 5,88^{b}$ & $1009,0 \pm 5,82^{c}$ & $1166 \pm 18,71^{d}$ \\
\hline \multicolumn{4}{c}{ Vacas con registro de cría } \\
\hline Cuartil 1 & Cuartil 2 & Cuartil 3 & Cuartil 4 \\
\hline $768,4 \pm 11,68^{a}$ & $895,5 \pm 4,58^{b}$ & $1009,0 \pm 6,52^{c}$ & $1250 \pm 20,97^{d}$ \\
\hline VP: $n=26$ vacas por grupo $[F=153,7 ; P<0,0001]$. \\
VRC: $n=39$ vacas por grupo $[F=263,9 ; P<0,0001]$. \\
Todos los valores corresponden a la media aritmética \pm error estándar. \\
a,b,c,d Valores con diferente letra difieren al menos al $0,05$.
\end{tabular}

cuartiles restantes (segundo cuartil - VP: 30 meses y VRC: 29 meses; tercer cuartil - VP y VRC: 33 meses y cuarto cuartil - VP: 38 meses y VRC: 41 meses al primer parto se encuentran progresivamente cada vez más lejos del valor de 24 meses considerado óptimo.

Los resultados de la Tabla 3 ponen en evidencia la ausencia de diferencias estadísticamente significati- vas $(p>0,05)$ entre cuartiles, en la producción de leche ajustada a 305 días, en ambos tipos de vacas. En ninguno de los dos casos la máxima producción coincidió con las vacas más precoces (Cuartil 1). En el caso de las VP la mayor producción se presenta en el Cuartil 2, mientras que en las VRC lo hace en el Cuartil 3.

Tabla 3. Producción de leche ajustada a 305 días en la primera lactancia de vacas con diferente potencial de producción discriminadas en cuartiles por su edad al primer parto.

\begin{tabular}{cccc}
\hline \multicolumn{4}{c}{ Vacas puras } \\
\hline Cuartil 1 & Cuartil 2 & Cuartil 3 & Cuartil 4 \\
\hline $5287 \pm 246,9$ & $6175 \pm 324,8$ & $5783 \pm 218,6$ & $6103 \pm 285,9$ \\
\hline \multicolumn{4}{c}{ Vacas con registro de cría } \\
\hline Cuartil 1 & Cuartil 2 & Cuartil 3 & Cuartil 4 \\
\hline $4887 \pm 195,3$ & $5137 \pm 156,2$ & $5426 \pm 186,4$ & $5086 \pm 158,3$ \\
\hline VP: $n=26$ vacas por grupo $[F=2,226 ; P=0,090]$. \\
VRC: $n=39$ vacas por grupo $[F=1,614 ; P=0,1883]$. \\
Todos los valores corresponden a la media aritmética \pm error estándar.
\end{tabular}

Tabla 4. Intervalo parto-concepción (días) de vacas lecheras con diferente potencial de producción, discriminadas en cuartiles por su edad al primer parto.

\begin{tabular}{cccc}
\hline \multicolumn{4}{c}{ Vacas puras } \\
\hline Cuartil 1 & Cuartil 2 & Cuartil 3 & Cuartil 4 \\
\hline $199,0 \pm 19,90$ & $189,5 \pm 15,01$ & $213,5 \pm 19,39$ & $182,8 \pm 18,47$ \\
\hline \multicolumn{4}{c}{ Vacas con registro de cría } \\
\hline Cuartil 1 & Cuartil 2 & Cuartil 3 & Cuartil 4 \\
\hline $217 \pm 16,69^{a}$ & $159,6 \pm 12,37^{b}$ & $161,2 \pm 13,50^{b}$ & $154,3 \pm 12,28^{b}$ \\
\hline VP: $n=26$ vacas por grupo $[F=0,529 ; P=0,664]$. \\
VRC: $n=39$ vacas por grupo $[F=4,531 ; P=0,0045]$. \\
\multicolumn{2}{l}{ Todos los valores corresponden a la media aritmética \pm error estándar. } \\
a,b Valores con diferente letra difieren al menos al $0,05$.
\end{tabular}


Los valores de la Tabla 4 muestran que, en el caso de las vacas puras, no existen diferencias significativas entre cuartiles en el valor promedio del intervalo parto- concepción. A excepción del Cuartil 1 , los valores del indicador en las vacas con registro de cría son menores a los observados en las vacas puras. En ambos grupos de vacas, e independientemente de su precocidad, los valores del intervalo parto-concepción están lejos del óptimo de 82 días requerido para obtener un parto al año.

Los resultados de la Tabla 5 muestran ausencia de diferencias significativas entre cuartiles de precoci- dad, en el número de partos de las vacas tanto puras como con registro de cría. Los valores del indicador de supervivencia en el sistema son similares para ambos grupos de vacas y ponen de manifiesto una reposición del $33 \%$ en la mayoría de los cuartiles. La Figura 1 muestra una correlación positiva y significativa $(r=0,346 ; P=0,0002)$, entre la edad al primer parto y la producción de leche en las vacas puras, asociación que no alcanzó significado estadístico $(r=0,078 ; P=0,282)$ en el caso de las vacas con registro de cría.

Tabla 5. Número de partos de vacas lecheras con diferente potencial de producción, discriminadas en cuartiles por su edad al primer parto.

\begin{tabular}{lccc}
\hline \multicolumn{3}{c}{ Vacas puras } \\
\hline Cuartil 1 & Cuartil 2 & Cuartil 3 & Cuartil 4 \\
\hline $3(2-5)$ & $2,5(2-4)$ & $3(2-5)$ & $3(1,5-5,5)$ \\
\hline \multicolumn{4}{c}{ Vacas con registro de cría } \\
\hline Cuartil 1 & Cuartil 2 & Cuartil 3 & Cuartil 4 \\
\hline $4(2-5)$ & $3(2-5)$ & $3(2-4)$ & $3(2-5)$ \\
\hline VP: $n=26$ vacas por grupo [Estadística de $K W=2,651 ; P=0,449]$. \\
VRC: $n=39$ vacas por grupo [Estadística de $K W=2,515 ; P=0,473]$. \\
Todos los valores corresponden a la mediana (rango intercuartílico).
\end{tabular}
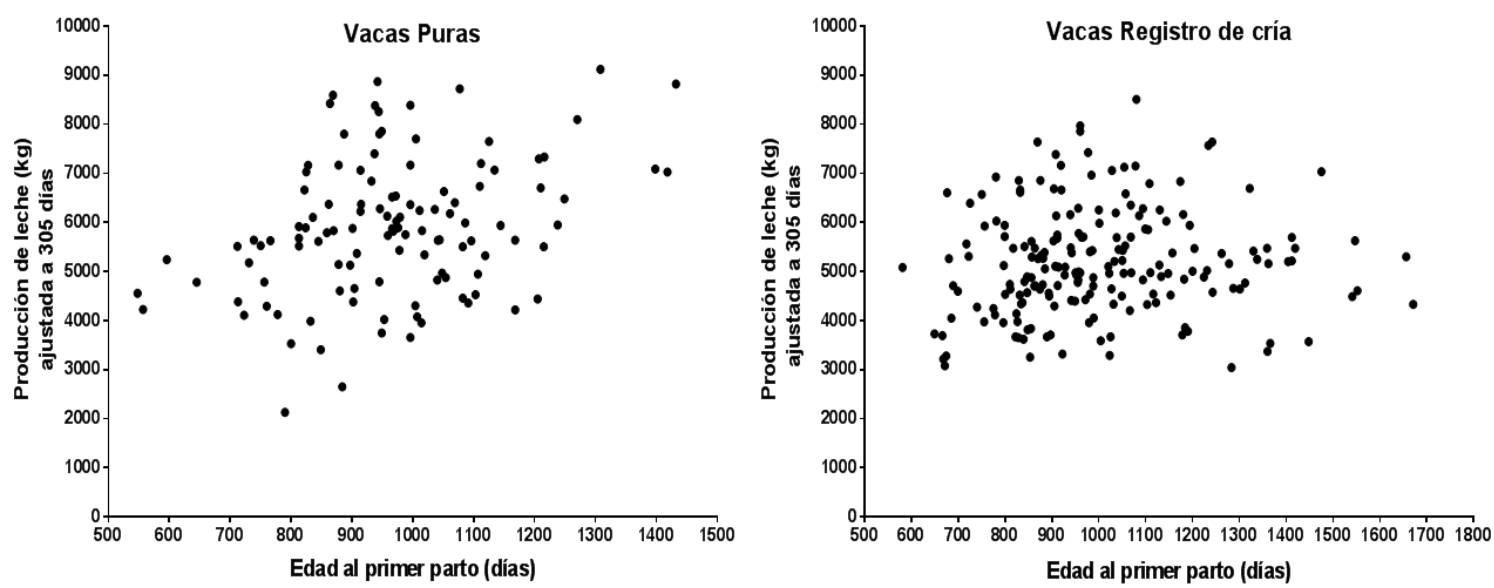

Figura 1. Asociación entre la edad al primer parto y la producción de leche ajustada a 305 días en dos grupos de vacas Holstein con diferente potencial de producción en sistemas a pastoreo.

La Figura 2 muestra ausencia de asociación $(r=$ $-0,093 ; P=0,353)$, entre la edad al primer parto y el intervalo parto-concepción en las vacas puras, mientras que en el caso de las vacas con registro de cría ambas variables se asocian en forma negativa $(r=-0,230)$ y estadísticamente significativa $(P=0,0028)$. 

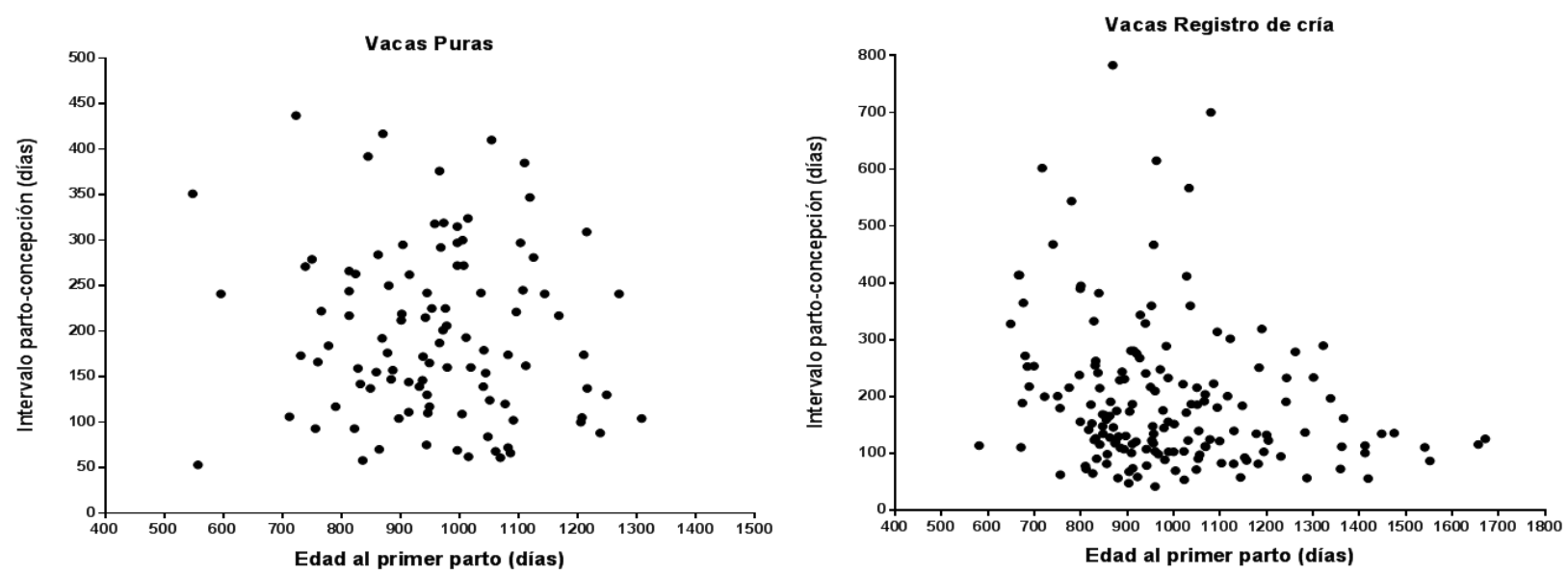

Figura 2. Asociación entre la edad al primer parto e intervalo parto-concepción en dos grupos de vacas Holstein con diferente potencial de producción en sistemas a pastoreo.
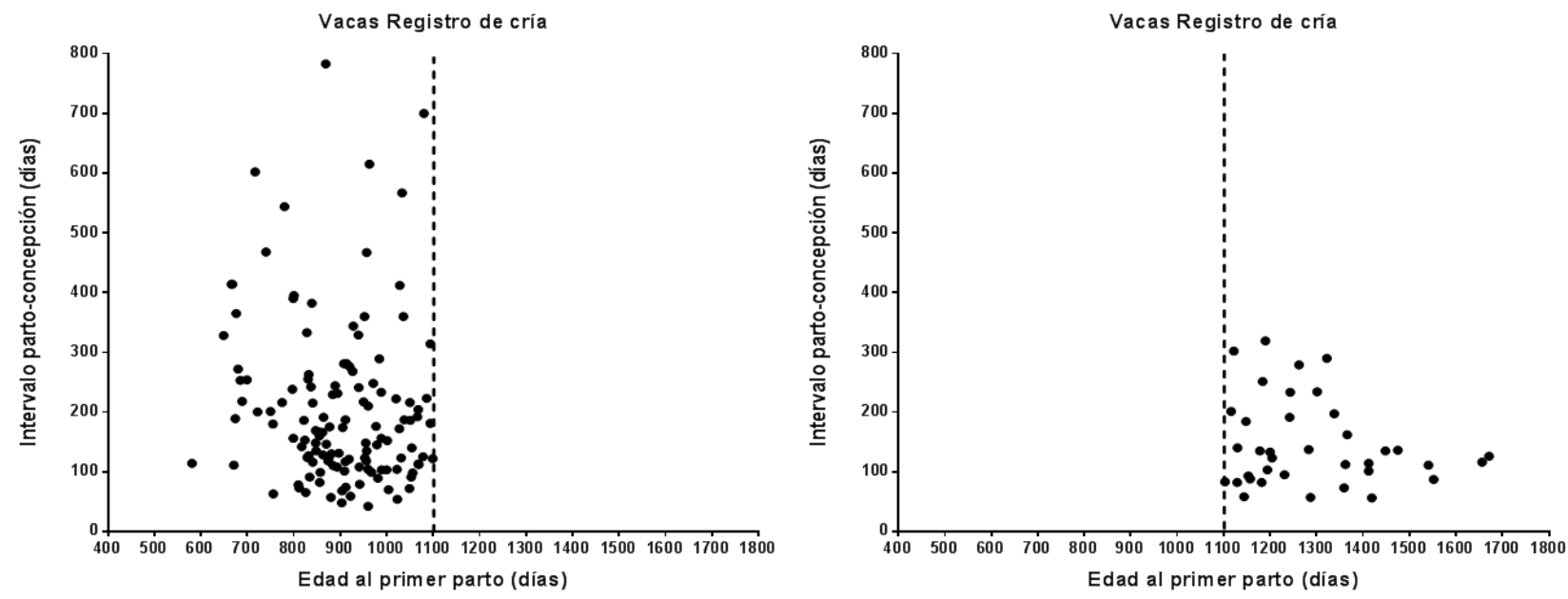

Figura 3. Asociación entre la edad al primer parto e intervalo parto-concepción en dos grupos $(<=1100$ días y $>=1100$ días $)$ e intervalo parto concepción de vacas Holstein con registro de cría en sistemas a pastoreo.

En el caso de las vacas con registro de cría, estas pueden identificarse dos grupos de animales (Figura 3); aquellos cuya edad al primer parto es menor a 1100 días $(\mathrm{n}=129)$ en los que la asociación es no significativa $(r=-0,130 ; P=0,141)$ y que muestra una gran dispersión en el valor del intervalo partoconcepción, y un segundo grupo, con edades al primer parto superiores a los 1100 días $(\mathrm{n}=37)$ en los que la asociación tampoco es estadísticamente sig- nificativa $(r=-0,189 ; P=0,263)$, pero los valores del intervalo parto-concepción muestran menor variación. El grupo de VCR con EPP $<1100$ días ( $n=$ 129) tiene valores de EPP: $900 \pm 115$ días y de IPC: $206 \pm 137$ días con un coeficiente de variación para IPC del 66,2\%. Por su parte, el Grupo de VRC con EPP >1100 días $(n=37)$ tiene valores de EPP: $1279 \pm 147$ días y de IPC: $147 \pm 73$ días con CV IPC $=49,8 \%$. 

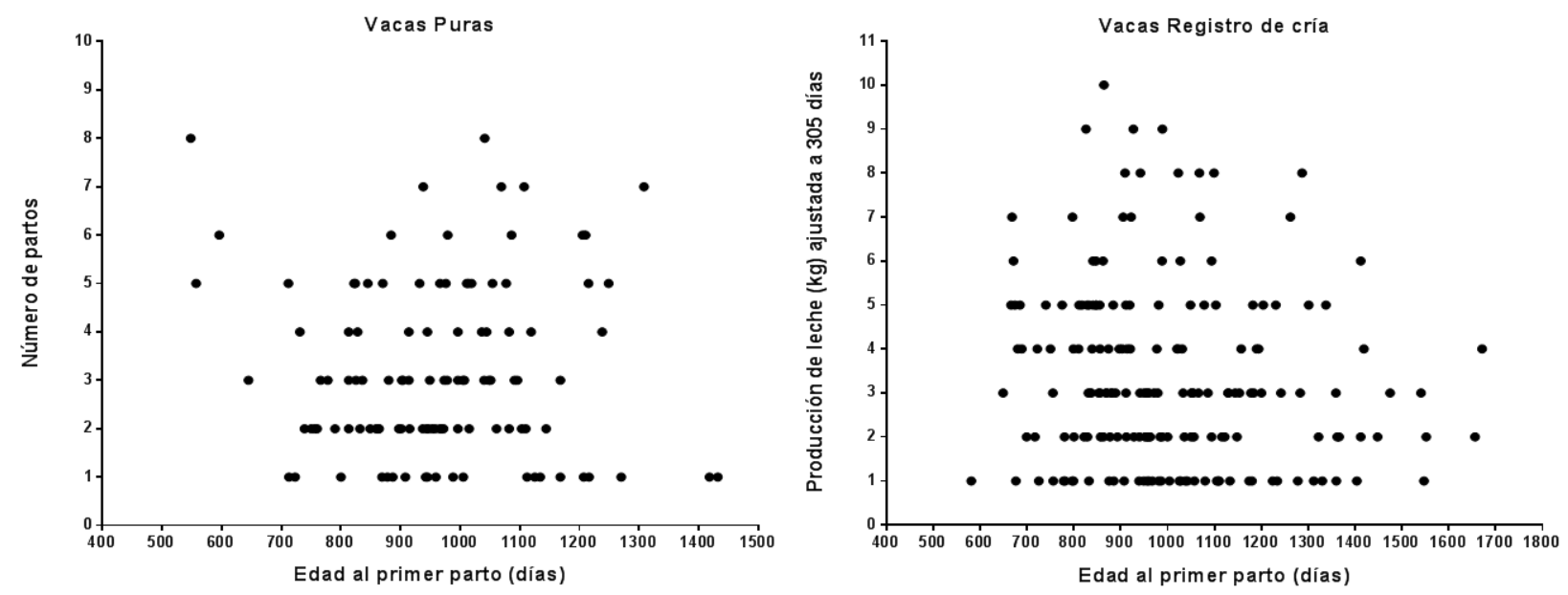

Figura 4. Asociación entre la edad al primer parto y número de partos en dos grupos de vacas Holstein con diferente potencial de producción en sistemas a pastoreo.

La Figura 4 resume el comportamiento del número de partos como indicador de supervivencia y la edad al primer parto. En las VP la asociación no fue estadísticamente significativa ( $\mathrm{r}$ de Spearman $\left.\left(r_{S}\right)=0,030 ; P=0,746\right)$, mientras que en el caso de las VRC la misma fue marginalmente significativa $\left(r_{S}=-0,138 ; P=0,057\right)$ y de signo negativo, es decir que, a mayor edad al primer parto menor número de partos.

\section{Discusión}

Los altos costos, la escala y la eficiencia de producción en los sistemas lecheros requieren en la actualidad un seguimiento riguroso de la vaquillona de reposición para asegurar el logro de márgenes aceptables de rentabilidad. La inversión del futuro, en este tipo de establecimientos, es la vaquillona de reposición. El manejo adecuado de la misma en las etapas de cría y recría, adaptado a cada sistema de producción y con un manejo sanitario programado según región, escala e intensificación del sistema son factores esenciales a considerar. Los dos grupos de vacas comparados en este trabajo no se diferenciaron en forma significativa en la edad promedio al primer parto, cuyo valor se encuentra muy por encima del óptimo deseable de 24 meses (Hare, Norman y Wright, 2006). Los valores relevados para esta variable son mayores que los informados por otros autores (Pirlo, Miglior y Speroni, 2000; Salazar-Carranza y col., 2013), pero simi- lares a los reportados por García Bouissou y Gens, (1997) y Snyder, (2006). Las producciones de leche de ambos grupos coinciden con los datos informados en algunas publicaciones (Glauber, 2007), aunque son menores a los 6500 litros y 7500 litros obtenidos por otros (Krupic y col., 2015; Romano, Salas y Maiztegui, 2016), a nivel local y para las mismas condiciones de manejo. Se observaron diferencias significativas en los valores promedio del intervalo parto-concepción; los mismos fueron superiores a los $133 \pm 9$ días promedio informados por Krupic y col., (2015), y mostraron una tendencia en las VRC a ser más fértiles que las VP.

En cuanto a la longitud de vida productiva no se observaron diferencias significativas, coincidiendo el número de partos con el número promedio de 2,6 a 3,3 pariciones, informado en vacas con alto rendimiento productivo en los Estados Unidos y Alemania (Hare, Norman y Wright, 2006; Knaus, 2009; Frana y col., 2014). La consideración conjunta de estas variables indicaría que, en promedio, estas vacas llegaron al primer parto a una edad avanzada con una moderada producción de leche, con dificultades para quedar preñadas luego del primer parto y con una disminución en la longitud de su vida productiva.

La categorización de ambos tipos de vacas en cuartiles de precocidad permitió comparar el desempeño de cuatro grupos de animales con diferencias en la edad al primer parto. En este sentido, los resultados muestran que sólo el $25 \%$ de las vaquillonas llegan a su primer parto a una edad cercana al ópti- 
mo de 24 meses, con una diferencia de $13-15$ meses entre las categorías extremas. Estos resultados, ponen en evidencia lo dificultoso que resulta cumplir en los sistemas a pastoreo con ciertos requisitos que son propios de otros sistemas productivos, sin una evaluación crítica previa de la factibilidad de llegar a la edad considerada óptima para el primer parto. En estos sistemas productivos puede verse afectado el tamaño del cuerpo y el inicio de la actividad hormonal del sistema reproductivo (Grajales, Hernández y Prieto, 2006). Indudablemente, en los sistemas a pastoreo existen factores ya sea desconocidos, o bien conocidos pero inmanejables en condiciones de manejo extensivo, que limitan de manera concluyente la posibilidad de cumplimentar etapas predeterminadas para sistemas productivos intensivos.

Heinrichs, (1993) sugirió para ganado Holstein una edad al primer parto de 23 a 24 meses. Gill y Allaire, (1976), por su parte, indicaron que para obtener un buen rendimiento durante toda la vida productiva el primer parto debería ocurrir entre los 22,5 a 23,5 meses. Estos objetivos, propios de otros ámbitos (sistemas intensivos), son los que se plantean para los sistemas a pastoreo. Sin embargo, García Bouissou y Gens, (1997) propuso, en base a diferentes opiniones, tanto de asesores como de productores, que la edad óptima al primer parto de vacas lecheras en sistemas a pastoreo debería tener un rango entre 22 y 27 meses de edad. Más allá que los sistemas de cría y recría se han modificado y se han intensificado desde el punto de vista de la alimentación, el límite superior de 27 meses del rango propuesto representa un valor más cercano a la realidad de los sistemas a pastoreo. Tal vez, el planteo para los sistemas a pastoreo, dista de la posibilidad real de cumplir con dicho objetivo. Duplessis y col., (2015) reportan que, en Quebec, el primer parto ocurre en promedio a los 27 meses, mientras que el objetivo es de 23 a 24,5 meses para maximizar la rentabilidad del rebaño.

La edad de las vaquillonas Holstein al primer parto generalmente varió entre los 24 y los 25 meses a lo largo de las últimas décadas (Mourits y col., 2000). Como el primer parto a los 24 meses de edad se está convirtiendo en un objetivo común y generalizado, es dable presuponer que la edad del primer parto continuará disminuyendo en el corto plazo. En estudios realizados con animales Jersey y Holstein de establecimientos especializados de Costa Rica (Castillo-Badilla y col., 2013; Salazar-Carranza y col., 2013) se determinó que la edad al primer parto ejerce una influencia significativa sobre los índices productivos y sobre la longevidad dentro del hato. Snyder, (2006) informó, en base a datos de la Asociación de Criadores de Holando Argentino, que la edad promedio al primer parto para las hembras de esta raza, para el período 1997 - 2007, era de 32 meses. Los sistemas lecheros en Argentina tienden a intensificarse, con un mayor confinamiento de los animales, y la búsqueda de un aumento de los litros de leche producidos por vaca con estabilización del consumo de materia seca (Frossasco y col., 2017). Sin embargo, en la llanura pampeana, el clima templado y las características de los suelos permiten disponer de una base forrajera de buena calidad a lo largo de todo el año, con los altibajos considerados propios de cada una de las estaciones (Molinuevo, 1998), apta para el desarrollo de sistemas de producción a pastoreo imponiendo, de alguna manera, un límite a las exigencias de crecimiento permanente en aras de cumplir objetivos productivos propios de otros sistemas.

Las vacas evaluadas en este trabajo no mostraron diferencias significativas entre cuartiles de precocidad en la producción de leche ajustada a 305 días. Ello implica que la edad a su primer parto no influyó en la producción de su primera lactancia. A este respecto cabe agregar que los valores de producción de leche están por debajo de los valores habituales de la zona y que la mayor producción no se registró en las vacas más precoces. En las puras esa mayor producción se dio en aquellas pertenecientes al Cuartil 2, mientras que en las vacas con registro de cría en el Cuartil 3, remarcando la discrepancia entre la precocidad pretendida y la precocidad recomendable para estos sistemas. Autores como Van Amburgh y col., (1994) y Hoffman y col., (1996), sugieren que la edad al parto puede ser reducida a los 22 meses sin influencias negativas en la producción de leche y la salud animal. Vitullo, Sarramone y Dick, (2016) no observaron asociación entre la edad al primer parto y la producción de leche, en coincidencia con lo informado en este trabajo para las vacas con registro de cría. Otros autores, por el contrario, concluyen que la producción de leche es mayor a medida que se atrasa la edad al parto (Castillo-Badilla y col., 2013; Salazar-Carranza y col., 2013), resultados que coinciden con lo encontrado en el caso de las vacas puras.

Lasa, (2015) observó una relación importante entre la producción de leche en la primera lactancia y la 
precocidad reproductiva, con un aumento de la producción con partos entre los 18 y hasta los 26 meses y un descenso posterior a medida que la edad al primer parto aumentaba. En relación con el reingreso de la vaca de primera parición a la actividad reproductiva, no se observaron diferencias significativas entre cuartiles de precocidad en el intervalo parto-concepción en el caso de las vacas puras, pero si en aquellas con registro de cría entre las más precoces -cuartil 1- y el resto de las categorías. Dicha diferencia indica que, para este grupo de vacas, un inicio temprano de la primera lactancia afecta el comportamiento reproductivo inmediato. El efecto de la edad al primer parto sobre los parámetros reproductivos, tales como servicio por concepción o intervalo parto-concepción, no han sido ampliamente discutidos en la literatura (Castillo-Badilla y col., 2016). No obstante, sí se ha relacionado la menor edad al primer parto con la presentación de partos distócicos, muy probablemente debido al escaso desarrollo corporal de las terneras (Ettema y Santos, 2004).

Por otro lado, se ha observado que la edad al primer parto pareciera no tener efecto sobre los indicadores reproductivos (Marini, Charmandarian y Di Masso, 2007), observación que se confirma en el grupo de vacas puras en el cual no hay asociación entre la edad al primer parto y el intervalo parto- concepción, contrariamente a lo encontrado por CastilloBadilla y col., (2016) que sí se observa en las vacas con registro de cría. En este último grupo, a mayor edad al primer parto menor intervalo partoconcepción. Algo interesante que mostró el grupo de vacas con registro de cría es que dentro del mismo pudieron identificarse dos grupos de animales, aquellos cuya edad al primer parto es menor a 36 meses en los que asociación es no significativa y muestra una gran dispersión en el valor del intervalo parto-concepción, y un segundo grupo, con edades al primer parto superiores a los 36 meses en los que la asociación tampoco fue estadísticamente significativa, pero los valores del intervalo partoconcepción muestran menor variación.

Este comportamiento puede explicarse si se toma en cuenta que luego del parto, los requerimientos del primer grupo incluyen producción, crecimiento y gestación, mientras que en los del segundo grupo sólo participan producción y gestación. Una de las posibles relaciones ventajosas de la edad al primer parto con las variables reproductivas es que su disminución podría aumentar el número de terneros por vaca, pero, tal disminución podría traer aparejada una mayor dificultad en el parto con la consecuente reducción en la viabilidad de los terneros y un aumento de su susceptibilidad a agentes infecciosos debido a una reducida absorción de inmunoglobulinas (Pirlo, Miglior y Speroni, 2000). La reducción de la edad al primer parto, además, conllevaría una reducción de la fertilidad, con aumento de los costos asociados a la reproducción, especialmente al intervalo entre partos y al descarte prematuro de animales valiosos (Moore y col., 1991). En contraste, la edad al primer parto no presentó efecto significativo sobre el intervalo parto concepción en vacas Jersey, coincidente con lo reportado para animales en condiciones de pastoreo, en donde no se observó efecto marcado de la edad al primer parto sobre los indicadores reproductivos (García Bouissou y Gens, 1997; Marini, Charmandarian y Di Masso, 2007). Otros autores, indican que las vaquillonas con mayor producción muestran un deterioro en su capacidad de reiniciar la actividad reproductiva luego del primer parto (Kim y Suh, 2003; Marini, Charmandarian y Di Masso, 2007).

De igual manera, Roche y col., (2013) asociaron alteraciones en el postparto con una alta producción de leche, especialmente en vacas que paren a edades entre 24 -28 meses y mayores a los 28 meses, lo cual podría indicar una alta predisposición de las vacas con una producción superior a presentar desordenes postparto. No se observaron diferencias significativas entre los cuartiles en el número de partos. Los valores de este indicador de longevidad fueron similares para ambos grupos de vacas, con una reposición del $33 \%$ en la mayoría de los cuartiles. En el caso de las vacas puras no se observó asociación entre la edad al primer parto y el número de partos mientras que, en aquellas con registro de cría, dicha asociación fue significativa $(p<=0,05)$ y mostró que a mayor edad al primer parto fue menor el número total de partos. El efecto de la edad al primer parto sobre la longitud de vida productiva tampoco ha sido ampliamente discutido, Casanova y col., (2011) analizaron el efecto de la edad al primer parto y observaron que vacas que parieron por primera vez a una edad avanzada -40 meses- presentaron un riesgo $23 \%$ superior de ser descartadas que vacas que lo hicieron a una edad de 30 meses. Sewalem, Kistemaker y Van Doormaal, (2003) observaron en la población Holstein de Canadá, que a medida que aumentaba la edad al primer parto se incrementaba el riego de eliminación. Castillo-Badilla y col., 
(2016) mostraron resultados confirmatorios de informes previos, según los cuales aquellas hembras con edad al primer parto menores a los 23 meses presentaron mayor longevidad dentro del hato en coincidencia con Cooke y col., (2013) y Zavadilová, Štípková y col., (2013). Vukasinovic, Moll y Casanova, (2001) informaron un mayor riesgo para las vacas que paren muy temprano y como así también, y especialmente, para las que paren muy tarde.

\section{Conclusión}

Las relaciones entre edad al primer parto con la producción láctea y con indicadores reproductivos y de longevidad en vacas lecheras de primera lactancia en sistemas a pastoreo resulta compleja; en tanto en este tipo de sistemas no es posible asegurar que los requerimientos de los animales serán cubiertos con el mismo grado de ajuste que se logra en sistemas intensivos. Las diferencias en el comportamiento de las vacas puras y las vacas con registro de cría con diferente potencialidad productiva, pone en discusión el objetivo de ejercer presión para lograr un primer parto a los 24 meses como propuesta generalizada en los sistemas a pastoreo.

\section{Referencias}

Berra, G. (1998). «Recría de vaquillonas en el tambo». En: Revista de Medicina Veterinaria 79.3, págs. 237-242.

Casanova, D. y col. (2011). «Análisis de la longevidad funcional dela razaHolando argentino». En: Sitio Argentino de Producción Animal 13.51, 21-29. Online:https://bit.ly/2EeKZ8X.

Castillo-Badilla, G. y col. (2016). «Efecto de la edad al primer parto sobre parámetros reproductivos en la primera lactancia de vacas Holstein y Jersey de Costa Rica». En: Ciencias Veterinarias 33.1, 33-45. Online:https://bit.ly/2EdCKKk.

Castillo-Badilla, Gloriana y col. (2013). «Efecto de la edad al primer parto sobre parámetros productivos en vacas Jersey de Costa Rica.» En: Agronomía Mesoamericana 24.1, 177-187. Online:https: / / bit.ly/2T21ddX.

Cooke, J. y col. (2013). «Association between growth rates, age at first calving and subsequent fertility, milk production and survival in HolsteinFriesian heifers». En: Open Journal of Animal
Sciences 3.1, 1-12. Oline:https : / / bit . ly / 2ByAX0H.

Duplessis, M. y col. (2015). «Weight, height, and relative-reliability indicators as a management tool for reducing age at first breeding and calving of dairy heifers». En: Journal of dairy science 98.3, 2063-2073. Online:https : / / bit . ly / 2WZUFeK.

Ettema, J.F. y J.E. Santos (2004). «Impact of age at calving on lactation, reproduction, health, and income in first-parity Holsteins on commercial farms». En: Journal of dairy science 87.8, 2730-2742. Online:https:/ / bit.ly /2GJBG2o.

Frana, E. y col. (2014). "Causas de descartes en vacas lecheras». En: XV Jornadas de Divulgación Técnico-Científicas. Ed. por Facultad de Ciencias Veterinarias. UNR, págs. 129-130.

Frossasco, G. y col. (2017). Evaluación de distintos sistemas lecheros intensivos. Inf. téc. Área Producción Animal EEA INTA Manfredi. Online: https: / / bit.ly/2GvCI2T.

García Bouissou, R. y M. Gens (1997). «Edad al primer parto en vaquillonas Holstein. Relaciones con el comportamiento productivo y reproductivo». En: Memorias del IV Simposio Lechero de Tandil. Ed. por Estudio Ganadero Pergamino. Argentina., págs. 33-38.

Gill, G.S. y F.R. Allaire (1976). «Relationship of age at first calving, days open, days dry, and herdlife to a profit function for dairy cattle». En: Journal of Dairy Science 59.6, 1131-1139. Online:https: //bit.ly/2V1Iqg8.

Glauber, C.E. (2007). «El manejo de la vaquillona de reposición en el rodeo lechero, una introducción. Revisión.» En: Veterinaria argentina 24.235, 366-370. Online:https://bit.ly/2SaA1VC.

Grajales, H., A. Hernández y E. Prieto (2006). «Edad y peso a la pubertad y su relación con la eficiencia reproductiva de grupos raciales bovinos en el trópico colombiano». En: Livestock Research for Rural Development 18.10, Online:https:/ / bit.ly / 2U7wVmS.

Grandl, Florian y col. (2016). «Biological implications of longevity in dairy cows: 1. Changes in feed intake, feeding behavior, and digestion with age». En: Journal of dairy science 99.5, 3457-3471. Online:https:/ / bit.ly /2toQhZa.

Hadley, G.L., C.A. Wolf y S.B. Harsh (2006). «Dairy cattle culling patterns, explanations, and implications». En: Journal of dairy science 89.6, 2286-2296. Online:https:/ / bit.ly/2BOSv95. 
Hare, E., H. Norman y J. Wright (2006). «Trends in calving ages and calving intervals for dairy cattle breeds in the United States». En: Journal of dairy science 89.1, 365-370. Online:https:/ / bit.ly/ 2BFe5wr.

Heinrichs, A.J. (1993). «Raising dairy replacements to meet the needs of the 21st century». En: Journal of Dairy Science 76.10, 3179-3187. Online:https:/ / bit.ly/2EciR6b.

Hoffman, P.C. y col. (1996). «Effect of accelerated postpubertal growth and early calving on lactation performance of primiparous Holstein heifers». En: Journal of dairy science 79.11, 2024-2031. Online:https://bit.ly/2TRO1p1.

Kim, I.H. y G.H. Suh (2003). «Effect of the amount of body condition loss from the dry to near calving periods on the subsequent body condition change, occurrence of postpartum diseases, metabolic parameters and reproductive performance in Holstein dairy cows». En: Theriogenology 60.8, 1445-1456. Oline:https:/ / bit.ly/2EeeJmf.

Knaus, Wilhelm (2009). «Dairy cows trapped between performance demands and adaptability». En: Journal of the Science of Food and Agriculture 89.7, 1107-1114. Online:https : / / bit . ly / 2BHWtA5.

Krupic, M.L. y col. (2015). «Hip height measurements, production and reproduction in adult dairy cows in Argentina grazing systems». En: Asian J Agric Food Sci 3, 109-112. Online:https: / / bit.ly/2SF7BYT.

Lasa, D. (2015). «Comportamiento productivo y reproductivo de la población de bovinos Holando Argentino en las cuencas lecheras de la República Argentina.» Tesina. pp:46. Facultad de Ciencias Veterinarias, UNCPBA.

Marini, P. R., A. Charmandarian y R. J. Di Masso (2007). «Desempeño productivo y reproductivo de vacas de diferentes edades al primer parto en sistemas a pastoreo». En: Sitio Argentino de Producción Animal. APPA-ALPA-, 1-4. Online:http: //www.produccion $\backslash$ bibrangedash animal.com. ar/.

Marini, P.R., A. Charmandarian y M.I. Oyarzabal (2001). "Indicadores productivos y reproductivos de vacas de diferentes edades al primer parto en sistemas a pastoreo». En: ALPA Com 9, págs. 345-348.

Molinuevo, H.A. (1998). «Selección de bovinos para sistemas de producción en pastoreo». En: Rev. Arg. Prod. Anim. Vol. 18. 3-4, págs. 227-245.
Moore, R.K. y col. (1991). «Relationships between age and body weight at calving and production in first lactation Ayrshires and Holsteins». En: Journal of Dairy Science 74.1, 269-278. Online:https:/ / bit.ly /2SR4rRn.

Mourits, M.C. y col. (2000). «Optimization of dairy heifer management decisions based on production conditions of Pennsylvania». En: Journal of dairy science 83.9, 1989-1997. Online:https:/ / bit. ly/2X5yFiy.

Novaira, B. y col. (2018). «Predicción del Comportamiento Productivo de Vacas Lecheras en Sistemas a Pastoreo.» En: 1 Congreso Internacional Ciencia y Tecnología Agropecuaria - Ecuador, págs. 187-189.

Orrego, Jorge, Alfredo Delgado y Luisa Echevarría (2003). «Vida productiva y principales causas de descarte de vacas Holstein en la cuenca de Lima». En: Revista de Investigaciones Veterinarias del Perú 14.1, 68-73. Online:https: / / bit.ly / 2IdwXIC.

Pieroni, G. A. (2014). Curso Producción Bovinos de Leche.Recría de vaquillonas en el tambo. Inf. téc. pp6. Online:https:/ / bit.ly/2SGzgZH: Vetifarma.

Pirlo, G., F. Miglior y M. Speroni (2000). «Effect of age at first calving on production traits and on difference between milk yield returns and rearing costs in Italian Holsteins». En: Journal of dairy science 83.3, 603-608. Online:https:/ / bit.ly/ 2X9qm5h.

Roche, John R. y col. (2013). «Assessing and managing body condition score for the prevention of metabolic disease in dairy cows». En: Veterinary Clinics: Food Animal Practice 29.2, 323-336. Online:https:/ / bit.ly/2GuI920.

Romano, G., M. S. Salas y J. A. Maiztegui (2016). «Efecto de la estación y número de parto sobre la producción total de leche a 305 días de lactancia, en vacas Holando Argentino». En: XVII Jornadas de Divulgación Técnico-Científicas. IV Jornada Latinoamericana. II Jornadas de Ciencia y Tecnología. I Reunión Transdisciplinaria en Ciencias Agropecuarias. Facultad de Ciencias Agrarias y Veterinarias - UNR, 511-512. Online:https: / / fveter.unr. edu.ar/jornadas/.

Salazar-Carranza, Mauren y col. (2013). «Edad al primer parto en vacas Holstein de lechería especializada en Costa Rica.» En: Agronomía Mesoamericana, 233-243. Online:https : / / bit . ly / 2TZR4va. 
Seegers, H. y col. (1998). «Reasons for culling in French Holstein cows». En: Preventive veterinary medicine 36.4, 257-271. Online:https: / / bit.ly / 2GtmxDc.

Sewalem, Asheber, G.J. Kistemaker y Brian Van Doormaal (2003). "Genetic analysis of herd life in Canadian dairy cattle on a lactation basis using the survival kit». En: Interbull Bulletin 31, 73. Online:https:/ / bit.ly/2GKI2yA.

Snyder, M. (2006). La recría de vaquillonas en el negocio del tambo. Producir XXI 14: 43-49. Online:www. produccion-animal.com.ar.

Van Amburgh, M.E. y col. (1994). «Effect of prepubertal growth rate in Holstein heifers on first lactation milk yield». En: J. Dairy Sci 77.Suppl 1, pág. 185.
Vitullo, M., C. G. Sarramone y A. Dick (2016). «Análisis de distintas variables productivas y reproductivas, en relación con la edad al parto en vaquillonas Holando Argentino (Holstein).» Tesina de grado. Online:https: / / bit.ly / 2TSIWwA. Facultad de Ciencias Veterinarias -UNCPBA-.

Vukasinovic, N., J. Moll y L. Casanova (2001). «Implementation of a routine genetic evaluation for longevity based on survival analysis techniques in dairy cattle populations in Switzerland». En: Journal of Dairy Science 84.9, 2073-2080. Online:https:/ / bit.ly/2GNeIrm.

Zavadilová, L., M. Štípková y col. (2013). «Effect of age at first calving on longevity and fertility traits for Holstein cattle». En: Czech J. Anim. Sci 58.2, 47-57. Online:https:/ / bit.ly/2TYxUGp. 